\title{
Exploring the QCD phase diagram via reweighting from isospin chemical potential
}

\author{
B. B. Brandt, F. Cuteri, G. Endrődi and S. Schmalzbauer*i \\ Institute for Theoretical Physics, Goethe University, Max-von-Laue-Strasse 1, 60438 Frankfurt \\ am Main, Germany \\ E-mail: \{brandt, cuteri, endrodi, schmalzbauer\} eitp.uni-frankfurt.de
}

\begin{abstract}
We investigate the QCD phase diagram for small values of baryon and strange quark chemical potentials from simulations at non-zero isospin chemical potential. Simulations at pure isospin chemical potential are not hindered by the sign problem and pion condensation can be observed for sufficiently large isospin chemical potentials. We study how the related phase boundary evolves with baryonic and strange chemical potentials via reweighting in quark chemical potentials and discuss our results. Furthermore, we propose and implement an alternative method to approach nonzero baryon (and strange quark) chemical potentials. This method involves simulations where physical quarks are paired with auxiliary quarks in unphysical "isospin" doublets and a decoupling of the auxiliary quarks by mass reweighting.
\end{abstract}

37th International Symposium on Lattice Field Theory - Lattice2019

16-22 June 2019

Wuhan, China

\footnotetext{
${ }^{*}$ Speaker.

${ }^{\dagger}$ The research has been funded by the DFG via the Emmy Noether Programme EN 1064/2-1. S.S. has also received support from HGS-HIRe for FAIR and the Stiftung Giersch.
} 


\section{Introduction}

Despite the theoretical and experimental efforts of the past two decades to study the phase diagram and the properties of QCD at finite quark densities, most of the parameter space is still uncharted when it comes to first principles results. The main reason for this is the complex action problem affecting QCD at non-zero baryon chemical potentials, prohibiting direct simulations of lattice QCD. Despite the effort to overcome this problem (see [1], for reviews), there is currently no reliable method to obtain results close to the pseudo-critical temperature at physical quark masses.

In the grand canonical ensemble, three-flavor QCD at finite quark density can be described in terms of baryon $\left(\mu_{B}=3\left[\mu_{u}+\mu_{d}\right] / 2\right)$, isospin $\left(\mu_{I}=\left[\mu_{u}-\mu_{d}\right] / 2\right)$ and strange $\left(\mu_{s}\right)$ chemical potentials. In most of the physical systems all three chemical potentials are non-zero, while the effects due to non-zero $\mu_{B}$ are usually expected to dominate. In contrast to QCD at non-zero $\mu_{B}$ (and $\left.\mu_{s}\right)$, QCD at pure isospin chemical potential, i.e., $\mu_{B}=\mu_{s}=0$ and $\mu_{I} \neq 0$, with mass degenerate light quarks has a real and positive fermionic weight factor and, thus, permits direct simulations. After the first pioneering studies [2, 3, 4], we have recently presented the first results for the continuum phase diagram, see Fig. 1, with physical quark masses in a realistic three-flavour setup [5], showing Bose-Einstein condensation (BEC) of charged pions at large $\mu_{I}$ and small temperatures [6] (see also Refs. [7] for further applications of simulations at non-zero $\mu_{I}$ ).

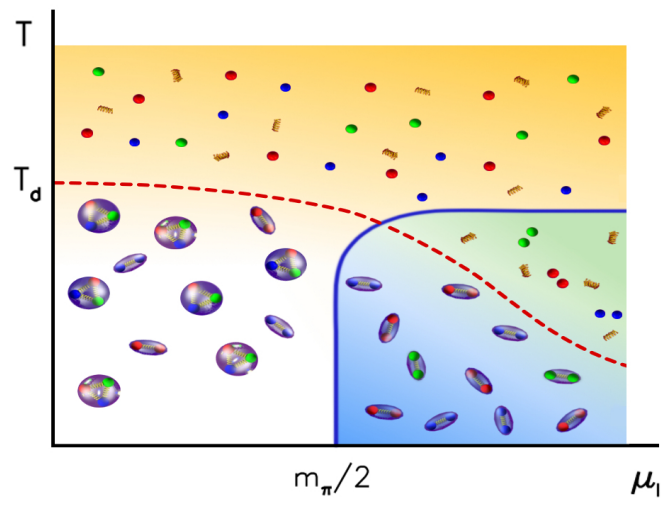

Figure 1: Conjectured phase diagram of QCD at pure isospin chemical potential (taken from [5]).

In general, it is the equation of state and the phase diagram at non-zero $\mu_{B}, \mu_{s}$ and $\mu_{I}$ which is of direct phenomenological importance. Since direct simulations are impossible, one is forced to use indirect methods, such as the Taylor expansion method and reweighting, to take the effects of non-zero $\mu_{B}$ and $\mu_{s}$ into account. These methods are restricted to small values of $\mu_{B}$ and $\mu_{s}$, rendering the important region beyond nucleon production threshold inaccessible. In this proceedings article, we apply the reweighting method to the region around the $\mu_{B}=\mu_{s}=0$ axis at zero temperature with the aim to trace the BEC phase boundary at non-zero $\mu_{B}$ and $\mu_{s}$. In particular, we test two different reweighting techniques for their efficacy. First, we use a reweighting in chemical potential (Sec. 3), starting from simulation points at non-zero $\mu_{I}$. Secondly, we present a novel technique (Sec. 4), which employs a reweighting in the quark mass to decouple auxiliary quarks (forming "isospin" doublets with the physical quarks) from the theory.

\section{Simulations at non-zero $\mu_{I}$}

The fermion matrix for a mass-degenerate fermion doublet of staggered quarks $a$ and $b$ at pure isospin chemical potential $\mu_{I}$ is given by

$$
\mathscr{M}_{a b}=\left(\begin{array}{cc}
\not D_{\mu_{I}}+m_{a b} & \lambda \eta_{5} \\
-\lambda \eta_{5} & \not D_{-\mu_{I}}+m_{a b}
\end{array}\right),
$$


where $\eta_{5}=(-1)^{n_{t}+n_{x}+n_{y}+n_{z}}$ and $\not D_{\mu}$ is the discretized massless Dirac operator at chemical potential $\mu$ and including stout smeared links. In QCD and our standard lattice setup and up to Sec. 4, we associate $a=u$ and $b=d$ and set $m_{a b}=m_{u d}$ to its physical value. We also include a physical strange quark at $\mu_{s}=0$ and use the Symanzik improved gluon action $S_{G}$. For $\mu_{I} \neq 0$, the invariance under chiral $\mathrm{SU}(2)_{V}$ rotations is broken down to a $\mathrm{U}(1)_{\tau_{3}}$ subgroup, which is spontaneously broken for $\mu_{I} \geq m_{\pi} / 2$ (in our convention for $\mu_{I}$, see [8]), leading to the condensation of charged pions. The unphysical off-diagonal terms in Eq. (2.1) break the residual $U(1)_{\tau_{3}}$ symmetry explicitly. The explicit breaking serves two main purposes: (i) it enables to observe spontaneous symmetry breaking in a finite volume; (ii) it acts as a regulator for lattice simulations in the BEC phase [2, 4]. Physical results are obtained in the limit $\lambda \rightarrow 0$.

Since simulations are done at $\lambda \neq 0$, i.e., including the symmetry breaking terms in Eq. (2.1), the full reweighting to the target ensemble includes reweighting factors $R_{\lambda}$ for the reweighting to $\lambda=0$. If $O$ is the observable evaluated with target ensemble parameters, the reweighted result is given by

$$
\langle O\rangle_{\lambda=0}^{\operatorname{targ}}=\frac{\left\langle O R^{\operatorname{targ}} R_{\lambda}\right\rangle_{\lambda}}{\left\langle R^{\operatorname{targ}} R_{\lambda}\right\rangle_{\lambda}}
$$

where $R^{\text {targ }}$ is the reweighting factor to target ensemble parameters. In practice, we will replace the reweighting factor $R_{\lambda}$ by its leading order expansion in $\lambda$, as implemented in the improvement program for the $\lambda$-extrapolation [8]. Consequently, there will remain a residual $\lambda$-dependence for the result on the left-hand-side of Eq. (2.2), which can be removed in terms of an extrapolation. Note, however, that the leading order reweighting typically captures most of the effects of the full reweighting factor, so that the effect of the remaining extrapolation is expected to be small compared to the effect of the reweighting to the target ensemble. Here we will focus on the reweighting to the target ensemble and skip the final $\lambda$-extrapolation for now.

\section{Reweighting in chemical potentials}

We first discuss the reweighting in chemical potentials in the vicinity of the $\mu_{B}=\mu_{s}=0$ axis. In particular, we are interested in the phase diagram and the behavior of the BEC phase boundary with $\mu_{B}$ and $\mu_{s}$. The reweighting strategy is sketched in Fig. 2. The ideal observable to study the BEC phase boundary would be the pion condensate, but its direct evaluation in the target ensemble at $\lambda=0$ is difficult. Instead we focus on the chiral condensate and the isospin density,

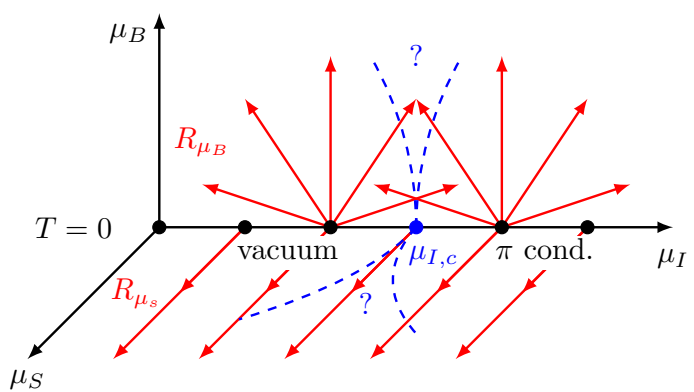

Figure 2: We simulate along the isospin direction and employ reweighting (red) to explore the BEC phase boundary (blue) in the vicinity of the $\mu_{B}=$ $\mu_{s}=0$ axis.

$$
\bar{\psi} \psi=\bar{u} u+\bar{d} d, \quad n_{I}=n_{u}-n_{d}, \quad \bar{q} q=\frac{T}{V} \frac{\partial \log \mathscr{Z}}{\partial m_{q}} \quad \text { and } \quad n_{q}=\frac{T}{V} \frac{\partial \log \mathscr{Z}}{\partial \mu_{q}} .
$$

At zero temperature, indications for the behavior of the BEC phase boundary can be obtained from lines of constant observables due to the Silver blaze phenomenon. 

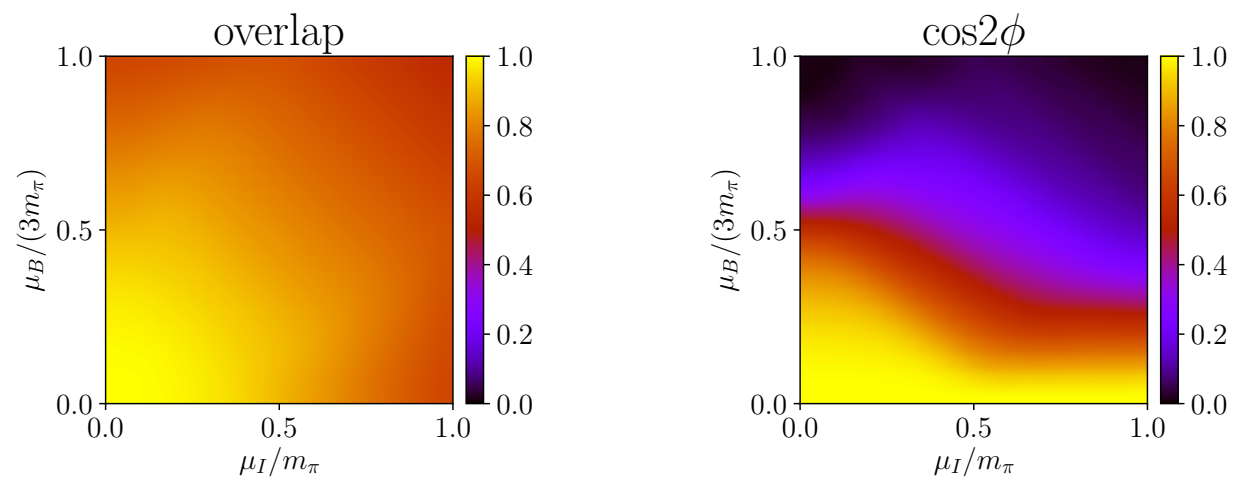

Figure 3: Overlap (left) and sign problem (right) obtained by reweighting from $\mu_{B}=\mu_{I}=0$.

\subsection{Reweighting setup}

The reweighting to the target ensemble is done by reweighting either of the fermion determinants in the partition function at $\lambda=0$ to a different value of $\mu_{q}$, starting from $\mu_{u}=-\mu_{d}=\mu_{I}$ and $\mu_{s}=0$. To reduce numerical costs, we use the determinant reduction formalism from [9, 10], where the reweighting factor from $\mu \rightarrow \mu^{\prime}$ is written as

$$
R^{\operatorname{targ}}\left(\mu^{\prime}, \mu\right)=\left(\frac{\operatorname{det} M\left(\mu^{\prime}\right)}{\operatorname{det} M(\mu)}\right)^{1 / 4}=e^{-3 V_{s} L_{t}\left(\mu^{\prime}-\mu\right) / 4} \prod_{i}\left(\frac{p_{i}-e^{L_{t} \mu^{\prime}}}{p_{i}-e^{L_{t} \mu}}\right)^{1 / 4},
$$

where the $p_{i}$ are the eigenvalues of a reduced matrix $P$, depending on $m_{q}, V_{s}$ is the spatial volume and $L_{t}$ is the temporal extent. Once the $p_{i}$ are known, the reweighting in $\mu$ is analytic and can be done for different values of $\mu^{\prime}$ at once. In practice, we employ the rooting to each individual term in the product of Eq. (3.2), selecting the complex roots which ensure that the determinant is real and positive at $\mu_{B}=\mu_{s}=0$ and continuous along the real $\mu_{B}$ or $\mu_{s}$ axis. All results presented in the following have been obtained on $8^{4}$ lattices.

The parameter range to which the reweighting method can be applied is limited by the overlap between initial and target ensembles and the severity of the sign problem. To estimate the overlap $\gamma$, we extend the argument from $[11,12]$ to complex reweighting factors. Considering a sorted set of reweighting factors $R_{i}\left(\left|R_{1}\right| \geq \ldots \geq\left|R_{N}\right|\right)$, which are normalized, $\sum_{i}\left|R_{i}\right|=1$, we define $\gamma$ implicitly via $\sum_{i=1}^{N \gamma / 2}\left|R_{i}\right|=1-\gamma / 2$. If $\gamma=1$ all configurations have similar weights, i.e., the overlap is large. If only a few configurations effectively contribute $\gamma$ will be close to zero and expectation values can be biased. The severeness of the sign problem can be assessed from the phase fluctuations of the reweighting factor $R=|R| e^{i \phi}, \cos (2 \phi)=\operatorname{Re} R^{2} /|R|^{2}$. Values close to unity indicate small phase fluctuations, whereas small values indicate a severe sign problem. Both quantities are shown for the reweighting from $\mu_{B}=\mu_{I}=0$ to $\mu_{B}, \mu_{I} \neq 0$ in Fig. 3. In the following we only present results with $\gamma>0.5$ and where the partition function is real and positive, another indication that the sign problem is under control.

\subsection{Results}

We will first discuss the results obtained at non-zero $\mu_{s}$. The reweighted results for the strange 

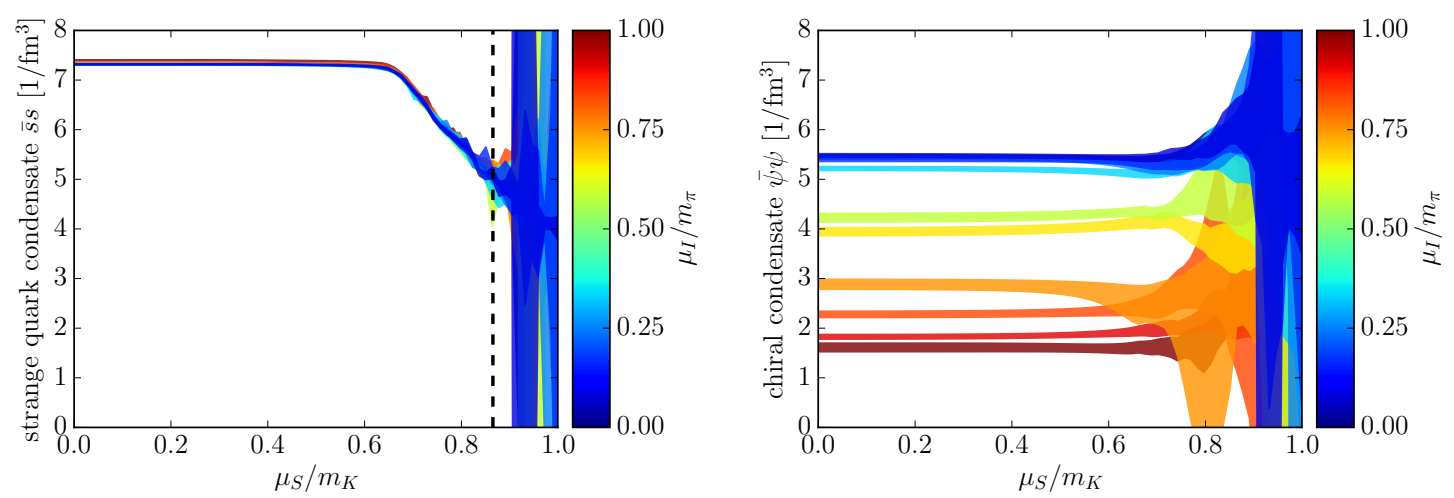

Figure 4: Effect of $\mu_{s}$ on the $\langle\bar{s} s\rangle$ (left) and $\langle\bar{\psi} \psi\rangle$ (right) for different values of $\mu_{I}$ (colour code). The dashed line on the left indicates the limit for kaon condensation [13].

quark and chiral condensates are displayed in Fig. 4. For all values of $\mu_{I}$ the strange quark condensate drops significantly for $\mu_{s}>0.6 \cdot m_{K}$, which could be an indication for a phase transition. The chiral condensate is mostly unaffected by $\mu_{s}$. In [13] it has been proposed that the onset of kaon condensation depends on $\mu_{I}$ and does not happen for $\mu_{S}<0.865 \cdot m_{K}$, indicated by the dashed line in Fig. 4. Unfortunately, reweighting looses its efficacy in this region. It might be that our signal is a precursor effect to kaon condensation due to finite size and temperature effects, but this will have to be clarified in the future. Below $\mu_{s}=0.6 \cdot m_{K}$ we clearly do not observe any effect of $\mu_{s}$ on the BEC phase boundary.

The results for the chiral condensate reweighted to non-zero $\mu_{B}$ are shown in Fig. 5. In our setup an indicator for the BEC phase boundary is a strong decrease of the chiral condensate. We observe that the surface of strong depletion of the condensate (the purple region) bends towards larger values of $\mu_{I}$ in Fig. 5 and we interpret this as an indicator for a similar bend of the BEC phase boundary. It is unexpected that the chiral condensate also shows a slight fall off across the line from $\left(\mu_{B}=0, \mu_{I}=\right.$ $\left.m_{\pi} / 2\right)$ to $\left(\mu_{B}=3 m_{\pi} / 2, m_{I}=0\right)$. Due to the Silver Blaze phenomenon one does not expect a depletion of the chiral condensate outside of the BEC phase up to $\mu_{B} \approx m_{N}$ (the mass of the nucleon). We are currently investigating the origin of this, likely unphysical, behavior.

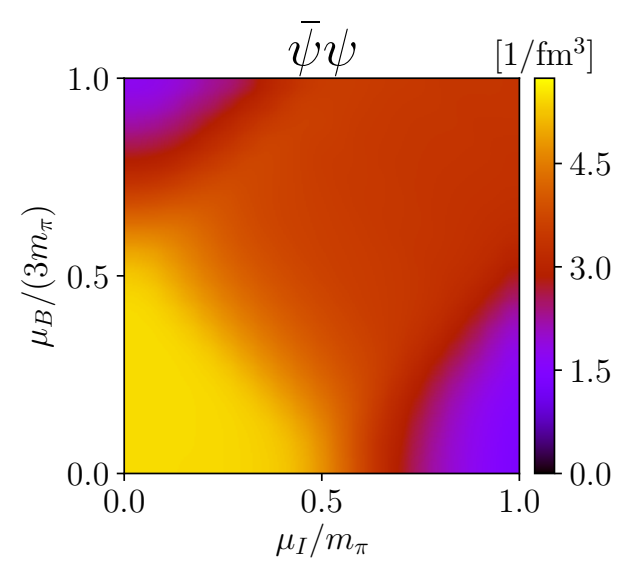

Figure 5: The chiral condensate at non-zero $\mu_{B}$. We combined several auxiliary ensembles in this plot, only considering the ones with sufficient overlap with the target ensemble and a reasonably controlled sign problem.

\section{Reweighting in quark masses}

In addition to reweighting in chemical potentials from finite $\mu_{I}$, we propose a novel reweighting direction to access the phase diagram. The phase diagram with $N_{f}=2+1$ dynamical flavours 
can, in principle, be accessed by simulating with a $N_{f}=2+2+1$ setup, including light quarks at the desired quark chemical potentials, accompanied by auxiliary "isospin" partners with negative chemical potential. The latter are decoupled from the theory by increasing their mass $m_{a}$ (initially $m_{a}=m_{u d}$, for instance) via reweighting and taking the limit $m_{a} \rightarrow$ $\infty$. The underlying idea and the possible phase diagram for different values of $m_{a}$ is depicted in Fig. 6.

The reweighting factor for altering one of the quark masses of the quarks in an isospin doublet, here the one with negative chemical potential, from $m_{u d}$ to $m_{a}$ is given by

$$
R^{\operatorname{targ}}=\left[\frac{\operatorname{det}\left(\not D_{-\mu_{I}}+m_{a}\right)}{\operatorname{det}\left(\not D_{-\mu_{I}}+m_{u d}\right)}\right]^{1 / 4} .
$$

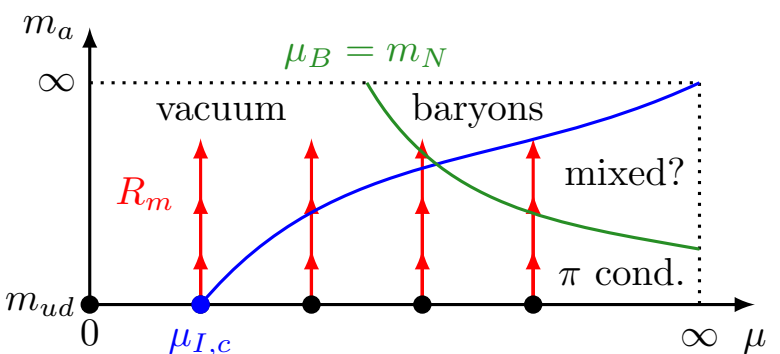

Figure 6: A possible scenario for how the quark masses could affect the phase diagram at finite quark chemical potential $\mu$. Changes in the auxiliary quark mass(es) influence both the BEC (blue) and baryon creation (green) phase boundaries. The red arrows indicate the reweighting direction.

Since $\not D_{-\mu_{I}}$ is a non-normal operator, the reweighting to different values of $m_{a}$ can again be done analytically when we know the left and right eigenvectors of $\not_{-\mu_{l}}$. The non-normality also needs to be taken into account when computing observables [14].

We observed that adding light quarks induces drastic changes in the system, strongly affecting the lattice spacing, $m_{\pi}$ and the value of $\bar{\psi} \psi$. To improve the overlap between initial and target ensembles, we tuned the masses of the isospin doublets in the Silver blaze region such that the values of $\bar{\psi} \psi$ roughly match those of the $N_{f}=2+1$ ensemble, making them about 11 times heavier. We then reweight the masses of the two physical quarks (the ones with positive chemical potential) to those of the target ensemble and decouple the auxiliary quarks by increasing $m_{a}$. Preliminary results for overlap and sign problem for such a reweighting are shown in Fig. 7. Apparently there is an optimal overlap between initial and target ensemble when varying $m_{a}$ by a factor between one to three. As expected, the sign problem reappears and becomes stronger with increasing $m_{a}$.
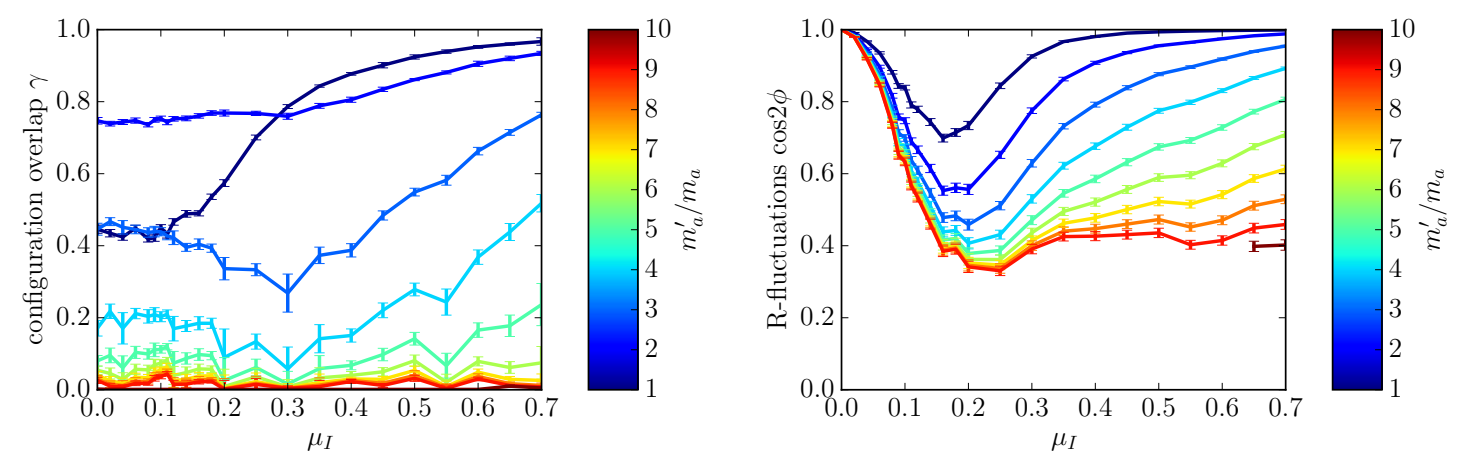

Figure 7: Overlap (left) and sign problem (right) in case of the mass reweighting described in the text. 


\section{Summary and Outlook}

In this proceedings article, we presented results for the pion condensation phase boundary for small baryonic and strange chemical potentials obtained from reweighting in chemical potentials from ensembles generated at finite isospin chemical potentials. The results are still in a preliminary stage and have been obtained on small $\left(8^{4}\right)$ lattices. We observed an unexpected behavior of the chiral condensate at non-zero baryon chemical potential and we are currently investigating possible causes. One issue concerns the ambiguity in evaluating the phase factor of the rooted reweighting factor of Eq. (3.2). A new method to avoid the rooting has been proposed recently [15] and we plan to test it in the future. In addition, we proposed a novel reweighting in the masses of auxiliary quarks to study QCD at finite quark chemical potentials and presented first tests of this method.

\section{References}

[1] S. Gupta, PoS LATTICE 2010 (2010) 007 [arXiv:1101.0109]; C. Gattringer, PoS LATTICE 2013 (2014) 002 [arXiv:1401.7788]; H. T. Ding, PoS LATTICE 2016 (2017) 022 [arXiv:1702.00151].

[2] J. B. Kogut and D. K. Sinclair, Phys. Rev. D 66 (2002) 014508 [hep-lat/0201017]; Phys. Rev. D 66 (2002) 034505 [hep-lat/0202028]; Phys. Rev. D 70 (2004) 094501 [hep-lat/0407027].

[3] P. de Forcrand, M. A. Stephanov and U. Wenger, PoS LATTICE 2007 (2007) 237 [arXiv:0711.0023]; W. Detmold, K. Orginos and Z. Shi, Phys. Rev. D 86 (2012) 054507 [arXiv:1205.4224]; P. Cea et al, Phys. Rev. D 85 (2012) 094512 [arXiv:1202.5700].

[4] G. Endrődi, Phys. Rev. D 90 (2014) no.9, 094501 [arXiv:1407.1216].

[5] B. B. Brandt, G. Endrődi and S. Schmalzbauer, Phys. Rev. D 97 (2018) no.5, 054514 [arXiv:1712.08190].

[6] D. T. Son and M. A. Stephanov, Phys. Rev. Lett. 86 (2001) 592 [hep-ph/0005225].

[7] B. B. Brandt, G. Endrődi and S. Schmalzbauer, PoS Confinement 2018 (2018) 260 [arXiv:1811.06004]; B. B. Brandt and G. Endrődi, PoS LATTICE 2016 (2016) 039 [arXiv:1611.06758]. B. B. Brandt et al, Phys. Rev. D 98 (2018) no.9, 094510 [arXiv:1802.06685]. B. B. Brandt and G. Endrődi, Phys. Rev. D 99 (2019) no.1, 014518 [arXiv:1810.11045].

[8] B. B. Brandt, G. Endrődi and S. Schmalzbauer, EPJ Web Conf. 175 (2018) 07020 [arXiv:1709.10487].

[9] D. Toussaint, Nucl. Phys. Proc. Suppl. 17 (1990) 248.

[10] Z. Fodor and S. D. Katz, JHEP 0203 (2002) 014 [hep-lat/0106002].

[11] F. Csikor et al, JHEP 0405 (2004) 046 [hep-lat/0401016].

[12] C. Schmidt, hep-lat/0408047.

[13] A. Mammarella and M. Mannarelli, Phys. Rev. D 92 (2015) no.8, 085025 [arXiv:1507.02934].

[14] T. Tanaka, Journal of Physics A 39 (2006), 7757 [quant-ph/0603075].

[15] M. Giordano et al, arXiv:1911.00043. 